\title{
Perspectivismo y verdad en sociología: Bourdieu y Giddens
}

\author{
Perspectivism AND tRUth in SOCIOlogy: BouRdieU ANd GIDDENS
}

Dr. Jorge Gibert Galassi (jorge.gibert@uv.cl) Facultad de Ciencias Económicas y Administrativas, Universidad de Valparaíso (Valparaíso, Chile)

\begin{abstract}
From the traditional point of view, power and truth are not related. Since 20th century there has been a social and historical breach in conventional assumptions about the relationship between power and truth. On one hand, it is generally accepted that scientific facts are considered artificial, in the sense of being constructed. This assumption neither implied any kind of fraud nor did it mean that they could be created ex-nihilo. But the interpretation of Kuhn as well as the subsequent developments of the Strong Programme, successfully postulates that truth is merely a social issue. Since then, it has been increasingly suspected that any objective or neutral description of society is too difficult, rendering it completely illusory. That is why it is necessary to ask again for the relation between the structures of truth and the social structures of power and what kind of impact they have on contemporary sociological theory. In this paper, it is given two examples of that kind of enterprise related to two major leading sociologists: Bourdieu and Giddens.
\end{abstract}

Key words: perspectivism, truth, sociological theory, Bourdieu, Giddens.

\section{Resumen}

Desde el punto de vista tradicional, no hay asociación entre poder y verdad. Pero desde el Siglo XX existió un quiebre social e histórico en las presuposiciones convencionales acerca de la relación entre poder y verdad. De un lado, en general se acepta que los hechos científicos son artificiales en el sentido que son construidos y se asume que esto no implica algún tipo de fraude ni significa que ellos son creados ex-nihilo. Pero la interpretación de Kuhn, así como también los desarrollos posteriores del Programa Fuerte, permite postular que la verdad es un asunto puramente social. Desde entonces, se ha sospechado cada vez más que cualquier descripción neutral u objetiva de la sociedad es tan difícil que llega a ser completamente ilusoria. Por eso, es necesario preguntarse nuevamente por la relación entre las estructuras de la verdad y las estructuras sociales del poder y qué tipo de impacto ellas tienen en la teoría sociológica contemporánea. En este trabajo, se discuten dos ejemplos de ese tipo de empeño, relacionado con las obras de los sociólogos Bourdieu y Giddens.

Palabras clave: perspectivismo, verdad, teoría sociológica, Bourdieu, Giddens. 


\section{Introducción}

Desde la visión decimonónica, poder y verdad están desvinculados. Pero desde 1968 hay un quiebre social e histórico en los presupuestos convencionales concernientes a la relación entre poder y verdad. Foucault planteaba que "la mecánica del poder no se analizaba nunca. Sólo se pudo comenzar ese trabajo a partir de 1968" (1999:46). Por otro lado, si bien desde Bachelard se sabía que los hechos científicos son artificiales en el sentido de construidos, ello no significaba fraude ni que éstos pudieran ser creados exnihilo. Pero la interpretación de La Estructura de las Revoluciones Científicas de Kuhn y el posterior desarrollo del Programa Fuerte postuló, con éxito, que la verdad es un asunto puramente social. Desde entonces, la sospecha que la descripción objetiva o neutral de la sociedad es demasiado difícil se ha ido agrandando, hasta volverla ilusoria. No estamos de acuerdo. Hay que volver a preguntarse cuál es el vínculo entre las estructuras de verdad y las estructuras sociales de poder y cómo ellas impactan la teoría sociológica contemporánea.

Desde los aportes de Merton en sociología de la ciencia, se podría pensar que la teoría de la sociedad dejó de ser ingenua respecto de los orígenes y usos sociales del conocimiento al vincular la construcción de la verdad científica con los poderes establecidos. Mayo de 1968 puso en jaque los poderes establecidos, consagrando el giro de los años sesenta, que consistió en consagrar la legitimidad de diversos puntos de vista, debido a la consideración que así como la verdad científica surge en el contexto del poder establecido (trasformado en doxa), así también hay otras verdades igualmente legítimas que están ocultas debido a un origen lejano al poder establecido. La verdad quedó definida por su pertenencia a un grupo y, por tanto, como relativa a un grupo. Es el denominado perspectivismo. La potencia del perspectivismo ha tenido un marco general: el descrédito de la creencia de que el conocimiento científico es la mejor forma que adopta la verdad.

El pluralismo que hoy exhibe la teoría sociológica se abrió paso desde un razonable "parcialismo", o sea, la idea que la sociedad es tan grande que sólo cabe su análisis en porciones de realidad. Esta idea es parecida a la idea de las teorías de rango medio de Robert K. Merton y en cierta medida fue producto del descrédito de la gran teoría a lo Parsons, a partir de la crítica de C. W. Mills y John Rex y la imposibilidad de que las técnicas estadísticas a lo Stouffer generaran explicaciones causalmente interesantes. Ello derivó hacia un estéril perspectivismo, o sea, la creencia que la construcción teórica es la postura de un individuo que expresa el punto de vista, el interés o la doxa de un colectivo. En medio del debate sobre el poder (Sartre, Foucault, Mills, Coser, Darhendorf y un largo etc., entre otros el Habermas de Conocimiento $e$ Interés), el pluralismo teórico trocó en perspectivismo epistémico, es decir, la postura que asocia el contenido de la teoría con los supuestos políticos del grupo que adhiere a esa teoría, cuyo éxito depende del grado de poder que tal grupo posee. Y aunque sea un hecho que la posición de poder de un grupo específico influye en las descripciones de la sociedad, simplemente por el hecho que no es posible una descripción desde fuera de la sociedad, la pregunta es: ¿queda por ello invalidada la empresa teórica? Otra manera de preguntar es: ¿puede el pluralismo ser entendido de un modo no relativista?

Obviamente, nuestra postura es que la situación actual en sociología no sólo está en relación con el desarrollo del perspectivismo y la hegemonía a nivel de las presuposiciones que el relativismo ha alcanzado en la teoría. También ha jugado un rol la idea que no es necesaria una ontología de lo social para describir la sociedad y que toda descripción parte de un criterio arbitrario del sistema de observación (Luhmann), la creciente necesidad de explicaciones holistas de la sociedad (que a falta de buena sociología, han sido provistas por una contraproducente filosofía social), y el olvido de la tradición analítica de la sociología, en parte debido a la brecha existente entre la gran promesa y los límites de los métodos de regresión en los 
grandes sondeos y a la constatación del carácter dependiente de los resultados estadísticos respecto a los supuestos para su interpretación.

Pero en virtud del propósito inicial, discutiré la relación entre perspectivismo y verdad en la teoría sociológica, con la convicción que existe una confusión entre pluralismo y perspectivismo, y que la disciplina es intrínsecamente pluralista en un sentido básico. Finalmente, haré un breve comentario sobre dos teorías recientes, la del campo y la de la estructuración. Con todo, las conclusiones son preliminares.

\section{Primer argumento}

Las teorías de la verdad desarrolladas por la filosofía analítica han sido en general desconocidas por los teóricos de las ciencias sociales. Salvo excepciones, como Habermas, la teoría ha seguido una suerte de deflacionismo del rol de la verdad como correspondencia y ha abrazado el construccionismo social. Un punto de vista pragmático ha insistido en la integración de conceptos y enunciados generalistas. Otro, ha desarrollado una suerte de convencionalismo, excesivamente abstracto y con escasa potencia explicativa. De hecho, uno de los resultados de estas dos tendencias es la creencia generalizada que cada perspectiva teórica es válida bajo los parámetros de sus axiomas iniciales, que no necesitan dialogar con las otras teorías y, ni siquiera con la realidad, puesto que todo es interpretación o relato. Por otro lado, el hecho de la heterogeneidad que adoptan los sistemas sociales reales, junto a la percepción y relatos (teorías) también variados que emergen de ella, ha permitido que este giro perspectivista sea suficientemente radical en la filosofía de las ciencias sociales que ha hecho dudar de la posibilidad de una ciencia de la sociedad.

Históricamente, sin embargo, la sub-disciplina llamada sociología de la ciencia se ha relacionado con el problema de la verdad de las teorías científicas, básicamente desde el punto de vista de las influencias sociales que dan origen y caracterizan las representaciones de las teorías y su aceptación como verdaderas. Luego, un fundamento epistémico fundamental para las ciencias sociales son las teorías de la sociología de la ciencia, usualmente etiquetadas como perspectivismo social. Estas teorías definen, en mayor o menor grado, la verdad como un producto construido por grupos sociales. La verdad está referida a la tradición y cultura del grupo, incluido el grupo de los científicos, y supone la idea que el grupo da por descontado que su particular visión de las cosas se corresponde con la verdad de ellas. Así, una proposición es verdadera para una comunidad si y sólo si hay un consenso entre los miembros de la comunidad para creer en esa proposición. Es la versión 'consensual' del perspectivismo social. La comunidad científica se caracteriza por un conjunto de valores comunes, normas y prácticas de lo que resultan creencias compartidas. La segunda versión indica que la verdad es poder. Luego, basta administrar poder para sacramentar verdades. En esta dimensión, una perspectiva social nace de una comunidad fortalecida por privilegios de poder o autoridad, como la Iglesia o los médicos. Esta visión de la verdad como una consecuencia política está asociada con el concepto de "regímenes de verdad" de Michel Foucault. El tercer caso del perspectivismo social nace de la tradición marxista, que enfatiza la generación de la verdad mediante la lucha contra el poder o la autoridad, de tal modo que la verdad es el producto de la revolución emancipadora del proletariado, obtenida tras desvelar el carácter alienado del capitalismo.

De ser ciertas estas tres versiones, se produce la paradoja: una especialidad (la sociología de la ciencia) lapida a la disciplina madre, la sociología. La verdad objetiva de la sociología cede a la subjetividad del grupo que la produce, los sociólogos. 
Sin embargo, aunque es tautológico afirmar que la producción de conocimiento tiene un origen social y se desarrolla más o menos al ritmo y melodía de la dinámica social, no puede ser solo esto, a menos que sustentemos la idea que todo conocimiento social es científico o verdadero. Es decir, la justificación es sólo en parte social e histórica. La ciencia tiene su armonía propia. Sin añadir un elemento extra, la transhistoricidad de las verdades científicas y la objetividad de los enunciados no serían posibles.

\section{Segundo argumento}

Trataré de mostrar a continuación algunas ideas de la brecha existente entre los postulados fundamentales de la sociología de la ciencia y una ciencia particular: la sociología (entendida como representante de la verdad formal sobre la sociedad). En especial, mencionaré dos planteamientos respecto a la dinámica del conocimiento científico y haré un breve contrapunto con las teorías perspectivistas de la verdad, para mostrar si es posible un uso de ellas en la disciplina y cómo.

En esta parte, vuelvo a la idea más autóctona y original del pensamiento social: que es posible contar con una única y distintiva teoría de la sociedad (¿qué otra cosa podrían haber querido los padres fundadores?), donde el progreso científico es tomado en serio y que, en esa empresa, es necesaria una teoría de la verdad... al menos como canon de objetividad.

Así, a partir de algunas ideas de Bourdieu y Giddens, trataré de discutir lo que podría constituir una teoría de la verdad que soporte una construcción teórica en sociología más poderosa y fructífera. Pero primero, postularé las tesis de las ciencias o teorías sociales que vengo a discutir. Ambas surgen de un contrapunto con las ciencias o teorías que se ocupan del mundo natural:

(1) Todas las afirmaciones teóricas en sociología son verdaderas (luego la incógnita es dilucidar en qué grado, en qué nivel, en qué perspectiva demográfica o trivial), porque "todos tienen razón (en la sociedad)".

(2) Hay verdades más neutrales y estables que otras, en el sentido que varían menos temporal y geográficamente. Dicho de otro modo, ciertas dinámicas sociales son escurridizas a la determinación conceptual y otras no, pudiendo estas últimas ser parte de teorías bastante consistentes.

Si todos los seres humanos tenemos una "experiencia de la sociedad" y si esa experiencia de la sociedad es, de suyo, un elemento de la constitución de la sociedad, entonces, efectivamente todos los puntos de vista de esa experiencia de la sociedad son verdaderos, en tanto reales. Es decir, el punto de vista, expresado en una representación, tiene una correspondencia con el mundo social empírico. Ahora bien, ¿significa esto que toda representación es válida? Creemos que sí, pero esto no garantiza su inclusión en teorías generales, sino como elementos anómalos. Por ejemplo, una particular representación de un individuo en el plano del arte, puede ser solitaria y en ese caso, anómala. Sólo cuenta como social cuando da origen a una posición social.

Eso nos lleva a otro punto: como toda experiencia es potencialmente colectiva (en el sentido que varios individuos adhieren al creador o productor del punto de vista) y genera una posición social, se podría decir que sin posición social no hay punto de vista. Y si ese punto de vista siempre es parcial, entonces, todos los puntos de vista se fundamentan en preferencias epistémicas que surgen de esa posición. 
Lo importante aquí es lo siguiente: si bien todos los puntos de vista son verdaderos, eso no significa que todas las preferencias epistémicas sean equivalentes. Es aquí donde prima la preferencia epistémica que busca la "modalidad de ser de las cosas" (descripciones y explicaciones generales o sustantivas) en vez de datos o accidentes aislados.

Precisamente, ambos postulados (lejos de olvidar el problema de la verdad en ciencias sociales) ubican el tema de la verdad en una de las prioridades principales de las ciencias sociales. Pero, ¿qué es lo que se debe justificar como verdadero si todos los puntos de vista lo son?

Creo que el desafío consiste en intentar la coherencia o la inteligibilidad de todos los puntos de vista. Pero esto no es una mera sumatoria. Hay que partir por la identificación de puntos de vista legítimos, esto es, sociales y que supongan mecanismos reales que permitan convencer mediante la experiencia. Explico.

(a) No todo punto de vista es social y, por tanto, no todo punto de vista posee el mérito de ser incluido en una teoría social como generalidad, aunque sí como anomalía o descripciones particularistas que "esperan su turno" para ser integradas (o bien, olvidadas por los teóricos del futuro).

(b) Para que un punto de vista pueda ser abstraído o idealizado, ya sea en una teoría o un modelo, debe poder ser explicado en su emergencia y desarrollo a la par con algún mecanismo social real (ejemplo, punto de vista femenino).

(c) La empatía es la posibilidad de ampliar el punto de vista y ello se logra mediante la experiencia (no es un experimento mental, en el sentido habitual). La experiencia social de los viajes, la literatura y el cine, y tantos otros medios, permiten suponer que la validación de un punto de vista es la experiencia en vez del consenso, artificial de suyo o pura expresión de interés político.

La consecuencia de lo anterior es que el pluralismo no surge de una instancia metodológica sino que de la naturaleza misma de la realidad social bajo estudio. Debido a ello, se ha postulado que la teoría social siempre es reflexiva y crítica.

En la perspectiva de mi insistencia en una única teoría de la realidad social, usaré dos argumentos asociados a la búsqueda de la verdad y la objetividad en sociología. Uno es de Bourdieu, donde ellas son alcanzadas mediante la figura del intelectual colectivo, y el otro de Giddens a través de la doble hermenéutica.

\section{Los argumentos de la teoría del campo y la teoría de la estructuración}

Para Bourdieu, la verdad, como una proposición válida de una vez para siempre, no existe en ciencia: "La producción de verdades científicas posee una sociogénesis, inscrita en el campo social específico (el científico), que ni siquiera es un proyecto consciente aunque puede parecerlo" (Bourdieu y Wacquant 1992:128-129).

Dicho telegráficamente, Bourdieu propone que la sociedad es un vasto espacio social, que se ordena según campos. Cada campo es un espacio de luchas por un tipo específico de capital, el que ordena el campo y se mezcla con otros tipos de capital. En el caso del campo científico, lo que está en juego es el monto de capital simbólico y académico, pero en el desarrollo del juego participan de manera secundaria -pero en ocasiones decisiva- otros tipos de capitales, como el escolar, cultural, económico, el político o el social. 
Cada "jugador" (científico o laboratorio) ocupa una posición y posee un estilo de juego -una práctica, entendida como habitus- en función del monto del capital específico del campo que posee, la composición del total de capitales y la trayectoria histórica al interior del campo y sus vínculos con otros campos.

Ello no impide, como plantea Webb, que cualquier sociólogo, incluso Bourdieu, que opera dentro del campo de la sociología, 'sabe' que una ciencia "espera de sus practicantes que sean capaces de producir verdades científicas y arrojar certezas" (Webb et. al. 2004:61). En ese sentido, la concepción de Bourdieu es bastante convencional y análoga a la de Durkheim en el sentido que observa el mundo como algo enteramente desconocido, pero se diferencia en que "constantemente verifica los límites de sus métodos y la influencia de su posición" (Webb et. al. 2004:74).

Las ciencias sociales para Bourdieu son necesariamente un "conocimiento del conocimiento" (Bourdieu y Wacquant 1992:127). Ahora bien, este 'conocimiento del conocimiento': ¿es verdadero? O puesto en otros términos: ¿existe una verdad del mundo social? En sus conclusiones, Bourdieu es prístino: "la verdad es la relatividad generalizada de los puntos de vista" (Bourdieu 2003:198). Luego, por muchas "leyes" descubiertas o "estudios" realizados, la verdad del mundo social siempre es un objetivo de unas dobles luchas, las del mundo social y las del campo científico sociológico, y la ciencia social, al ser objeto y sujeto de vivencia, sufre un boicot incesante (e interminable) que siempre está frenando su autonomía.

Bourdieu se piensa entre dos ilusiones: la del sabio como mera expresión de una opinión (relativa) y la del Dios de Leibniz "el centro geométrico de todas las perspectivas" (lo absoluto). A esto último lo llama campo científico, espacio social que construye la verdad en una lucha que los innovadores ganan si y sólo sí conservan los capitales simbólicos que reglan la dinámica del campo, en una suerte de transición lenta de una verdad a otra según procedimientos regulados, y que se integran de modo progresivo gracias a la confrontación racional. Bourdieu es una posición que -sin renegar de ser sólo eso, una posición- forma parte del campo y sabe que el campo relacional es la realidad de su posición.

Esta postura no niega el progreso científico y aspira a un incremento de la objetividad. Creador y director del Centro de Sociología Europea, supo que la vigilancia socio-epistémica es insuficiente como atributo individual, ya que "la reflexividad sólo alcanza toda su eficacia cuando se encarna en unos colectivos que la han incorporado hasta el punto de practicarla de modo reflejo. En un grupo de investigación de esta índole, la censura colectiva es muy poderosa, pero es una censura liberadora, que hace pensar en la de un campo idealmente constituido, que liberaría a cada uno de los participantes de los 'sesgos' vinculados a su posición y a sus disposiciones" (Bourdieu 2003:195).

El principio del método científico denominado por Bourdieu 'intelectual colectivo' es una de las contribuciones más importantes, según sus adherentes. La creencia colectiva en que la teoría de Bourdieu era la 'correcta' condujo al reclutamiento de muchos jóvenes y no tan jóvenes investigadores que se unieron a su recién creado Centro de Sociología Europea, bajo la forma de una militancia intelectual. Bourdieu era una suerte de coach o director de orquesta que proveía "todos los estímulos en todos los niveles de la investigación...era el estratega y el entrenador, así como el capitán del campo" (Lenoir 2006:29). La creación de su propia doxa y su propio habitus científico fueron a la vez fuente de capital simbólico y académico que le permitieron posicionarse en el campo sociológico europeo.

Respecto a la doxa, ¿es esta portadora de alguna verdad distinguible de una falsedad? Me temo que nuestra duda respecto de la interpretación correcta de la doxa, es que ella se vuelve finalmente una propiedad holística, perdiendo así Bourdieu la oportunidad de enfrentar más decididamente la demanda 
por una 'verdad' que todo realista podría reclamar, o bien, ceder (de una buena vez) a las tentaciones relativistas que, por otro lado, esquiva. Por otro lado, Rogers Brubaker examina el habitus sociológico de Bourdieu y concluye que las características más importantes se derivan de sus conceptos centrales campo y habitus. Por este último, Brubaker señala: "disposición [de Bourdieu] a pensar en términos de disposiciones" (1993:220); polares o de oposiciones y, lo más interesante, su disposición a trascender estas polaridades. En el caso del campo, disposición a pensar en términos de unidades no societarias, sino espacios restringidos gobernados por lógicas propias, microcosmos sociales relativamente autónomos; pensar en términos topológicos, sistemáticamente (y agregaría relacionalmente), pensar comparativamente; pensar en términos de los intereses de cada práctica social; y finalmente, de pensar en capturar la lógica específica de campos específicos. Pensar relacionalmente solo es posible a través del tiempo, en la medida que tengo tiempo de reconstruir la red, de completarla, nunca sólo, acompañado de otros puntos de vista que la dialogan con mi punto de vista y, en ese sentido, es compatible con la 'verdad en el pasado' que sustenta la historia de la ciencia... y podría servir de base a una razonable filosofía de la ciencia.

Si nos remitimos a la teoría de Giddens, la investigación científica y el producto (el conocimiento científico) puede englobarse como práctica, donde nuestra interpretación es que toda práctica social es generada por reglas: las de la racionalidad científica. También recursos: el conocimiento científico (que incluye las tecnologías de observación, como la experimentación y la medición).

Una de las características de la teoría de Giddens es que rechaza concebir la conducta humana independientemente de la comprensión que los actores tienen de ella. Es en este sentido crítico de la visión naturalista de la sociología así como también rechaza el funcionalismo. Ambos elementos configuran lo que denominó el consenso ortodoxo, cuya crítica da inicio a la renovación ecléctica que él propone. Giddens enfatiza una concepción ontológica más que epistemológica de la teoría. Desechando la dicotomía funcionalismo/estructuralismo versus comprensivismo, propone estudiar "la conducta de los actores individuales que reproduce las propiedades estructurales de colectividades mayores" (1995:60). Y la respuesta, según la lúcida interpretación de Gaeta, Gentile y Lucero, es "el análisis de las prácticas de los actores sociales, actividades que se localizan en un espacio y un tiempo, y a través de las cuales los individuos reiteran las situaciones que las hacen posibles" (2007:289).

La sociología no es naturalista, pues se ocupa de lo que es constituido y producido por los actos de los sujetos. Así, la producción y reproducción de los sistemas sociales debe ser estudiada como parte de una actuación calificada por parte de sus actores. Siguiendo a Marx, afirma que los hombres producen la sociedad pero lo hacen como actores históricamente situados. Desde ahí, él avanza a la idea de que las sociedades que les toca vivir a los actores no coinciden necesariamente con las fronteras y dinámicas del Estado Nación o el mercado. Más bien son sistemas sociales localizados en un tiempo y lugar que poseen ciertas propiedades estructurales. En la modernidad, tales propiedades pueden ser bastante genéricas, debido a que existe un distanciamiento entre espacio y tiempo, que impiden a los actores estar "anclados" en la tradición per se y por tanto a desarrollar mecanismos de anclaje o seguridad ontológica en tanto seres humanos y, para ello, deben apropiarse reflexivamente del conocimiento disponible. Entonces, "en condiciones de modernidad el futuro se presenta siempre abierto, no sólo en términos de las corrientes contingencias de las cosas, sino también en términos de la reflexividad del conocimiento en relación al cual las prácticas sociales están organizadas" (Giddens 1990:84). La velocidad de los cambios, y la extensión de ellos, hacen que sea difícil establecer la verdad de los enunciados sociológicos, ya que la observación sociológica en ningún caso es un procedimiento automático. 
Lo que postula Giddens como mecanismo correctivo de manera permanente a la incertidumbre que hoy agobia a la teoría es su concepto de doble hermenéutica. El fundamento de esto es epistémico y ontológico, y se vincula con el hecho de que como los actores poseen capacidad cognoscitiva, la tarea de la sociología difiere del objetivo de las ciencias naturales. Los sociólogos deben interpretar un mundo social que es interpretado por los actores que en él habitan. La circularidad del proceso de conocimiento objetivo es compleja, pues en primer lugar no otorga preeminencia a una determinada episteme (científica versus cotidiana) y en segundo lugar ¿cuándo parar para no caer en una regresión infinita? Lo que sí está claro es que algunos conceptos sociológicos se filtran en el mundo cotidiano y cambian la manera de pensar de los actores cotidianos, como en el caso del concepto de rol o "el estilo de vida". Giddens plantea que es debido a esta doble hermenéutica que las explicaciones sociológicas deben ser sensibles a la auto comprensión y conocimiento de los actores en determinados lugares y tiempos históricos.

Las propiedades estructurales de los sistemas sociales generan constricciones pero también dan pie o permiten ciertas prácticas y no otras. Es la tesis de "la dualidad de la estructura" que indica brevemente que las estructuras pueden ser analizadas en términos de la estructuración, como una secuencia de prácticas reiteradas que permiten su reproducción. Es decir, cómo las estructuras (reglas y recursos organizados como propiedades estructurales) son constituidas por la acción social intencional y, recíprocamente, cómo la acción es constituida estructuralmente. Pero al hablar de estructuración, condición de la reproducción sistémica, Giddens plantea dos formas: una bajo condiciones que gobiernen la transformación del sistema o, la otra, bajo condiciones de mantención del sistema.

Ahora bien, el sistema social de la ciencia, según los lineamientos de Giddens, debería tener sus propios "procesos de estructuración donde interactúan significados (interpretaciones), normas y poder. Estos tres conceptos están implicados en las nociones de acción intencional y estructura: todo orden moral y cognitivo es al mismo tiempo un sistema de poder, que implica un horizonte de legitimidad" (Calhoun 1993:229).

Pero aquí pensamos que hay cierta ambigüedad en su pensamiento, pues, si es así, entonces, al menos las normas de los actores del sistema científico deberían ser sustancialmente distintas a las que ocupan los otros actores en la sociedad. Pero Giddens rechaza esta conclusión, planteando que no existe el observador privilegiado, sino que la posición del sociólogo no difiere de la de otros miembros de la sociedad. El entendimiento de ambos, sociólogo y persona común, es mutuo, en el sentido que sus esquemas interpretativos, que ambos usan, otorgan sentido a las actividades sociales y permite una caracterización reconocible de ellas. El científico social no puede disponer de la vida social como un "fenómeno" para observar independientemente de la representación del conocimiento que tiene de él, como un recurso mediante el cual lo constituye como "objeto de investigación". La inmersión en la vida social es la apuesta de Giddens para no caer en una actividad teórica sesgada. Para Giddens, lo anterior dista de un postulado metodológico, sino que es la misma condición ontológica de la vida en sociedad como tal.

\section{Conclusiones}

Existe complementación entre ambas teorías y aspectos en común. El primer complemento es la idea que la influencia "electromagnética" del campo de Bourdieu versus la reflexividad sistémica de Giddens, son dos momentos de un continuo. Es indudable que la influencia de factores objetivos del campo (dinero, tecnología, prestigio de los grandes centros) altera la producción y forma de las verdades. Pero también la reflexividad de un mundo globalizado lo hace. 
También una tesis común es que la realidad social existe en las cosas y en los cuerpos. Es una versión más naturalizada de la vieja tesis que lo social existe fuera del individuo y dentro de él, en su mente o algún componente "indeterminado" y constituye el fundamento para obviar la dicotomía estructura/agente. Las estructuras sociales externas son posibles si hay mecanismos que permitan su reproducción, y estos mecanismos deben funcionar, ser movilizados, por cuerpos cuya característica es poseer "estructuras sociales internalizadas".

Ambos autores son claros respecto al hecho que la sociología, al tener por objeto al mundo social, no puede reclamar su objeto de modo categórico, pues todos quieren participar y producir una representación legítima (científica o incluso "mejor de la ciencia", como los periodistas que abogan por la primacía del reporte verídico "en terreno"). Luego, desde Bourdieu, en el campo son menos categóricas las diferencias entre productores y consumidores, y los científicos sociales están más a merced del capital simbólico de los medios de comunicación, que por su sola masividad, imponen una visión de la realidad social. Por su parte, Giddens alegaría que los sociólogos deberían involucrarse más en las tramas de poder y generar mayor reflexividad que los agentes cotidianos para "ganar" esos espacios.

En Giddens y Bourdieu, el status científico de las ciencias sociales está en entredicho. Si bien se afirma la cientificidad de la sociología, se deja en claro que no es posible una justificación puramente epistémica. En nuestra postura, por el contrario, creemos que ambos autores fundamentaron externamente la producción y exactitud de los enunciados desde el punto de vista social e histórico, pero no se atrevieron a declararse realistas y menos a defender una teoría de "la verdad" como correspondencia.

\section{Nota}

Este artículo fue concebido en el marco del Proyecto Fondecyt No. 11060031 "Coherentismo o fundacionalismo: estudio sobre la justificación epistémica (evidencia) en las teorías sociológicas de Pierre Bourdieu y Anthony Giddens".

\section{Bibliografía}

Bourdieu, P. 2003. Ciencia de la ciencia y reflexividad. Barcelona: Anagrama.

Bourdieu, P. y Wacquant, L. 1992. An invitation to reflexive sociology. Chicago: The University of Chicago Press.

Brubaker, R. 1993. Social theory as habitus. En: C. Calhoun et. al. Bourdieu: critical perspectives. Chicago: The University of Chicago Press, pp. 212-234.

Calhoun, C. et. al. 1993. Bourdieu: critical perspectives. Chicago: The University of Chicago Press.

Foucault, M. 1999. Estrategias de poder. Obras esenciales, volumen II. Barcelona: Paidós.

Gaeta, R; Gentile, N. y Lucero, S. 2007. Aspectos críticos de las ciencias sociales. Entre la realidad y la metafísica. Buenos Aires: Eudeba. 
Giddens, A. 1995. La constitución de la sociedad. Bases para la teoría de la estructuración. Buenos Aires: Amorrortu Editores.

Giddens, A. 1990. Consecuencias de la modernidad. Madrid: Alianza editorial.

Lenoir, R. 2006. Scientific habitus. Pierre Bourdieu and the collective intellectual. Theory, culture \& society 23(6): 25-43. doi: 10.1177/0263276406069774

Webb, J; Schirato, T. y Danaher, G. 2004. Understanding Bourdieu. London: Sage.

Recibido el 5 Sep 2014

Aceptado el 23 Nov 2014 